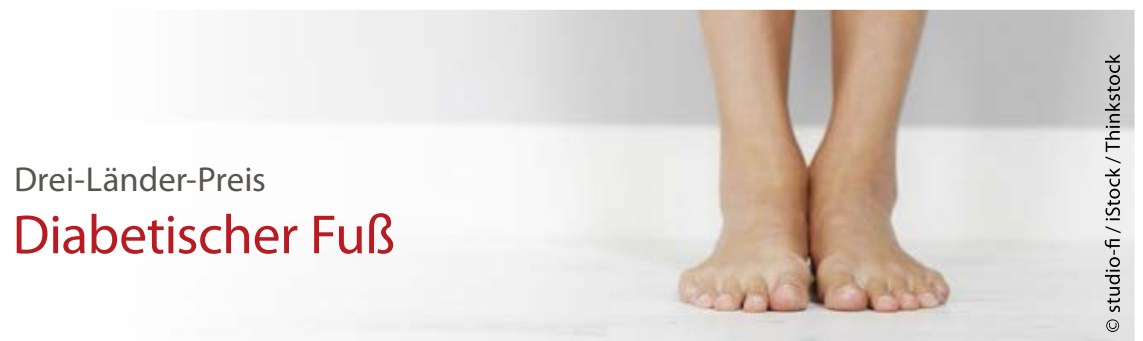

- Das Diabetische Fußsyndrom ist eine sehr schwerwiegende Erkrankung, die wegen der Komplexität des Krankheitsverlaufs ein Problem in der medizinischen Versorgung darstellt. Mit dem Ziel, innovative Therapiemaßnahmen zu finden und auch eine verstärkte multidisziplinäre Zusammenarbeit der medizinischen und pflegerischen Fachkräfte bei der Behandlung und Versorgung dieser Patienten zu fördern, schreibt die gemeinnützige Stiftung Fondation URGO zum zweiten Mal den Drei-Länder-Preis Diabetischer Fuß in Deutschland, Österreich und der Schweiz (DACH) aus.
Examinierte Pflegefachkräfte, Pflegewissenschaftler sowie Ärztinnen und Ärzte aller Fachgebiete einschließlich Doktoranden der Medizin können ihre Arbeiten zur Prävention und zum Management des Diabetischen Fußsyndroms bis zum 31.12.2015 elektronisch per Mail einreichen unter: dfupreis2015@urgo.de

Alle eingereichten Arbeiten werden von einem unabhängigen, nationalen Expertengremium gesichtet und begutachtet. Der Preis ist mit insgesamt $20.000 €$ dotiert.

www.urgo.de.

\title{
Vorreiter Rheinland-Pfalz
}

\section{Modellprojekt Gemeindeschwesterplus}

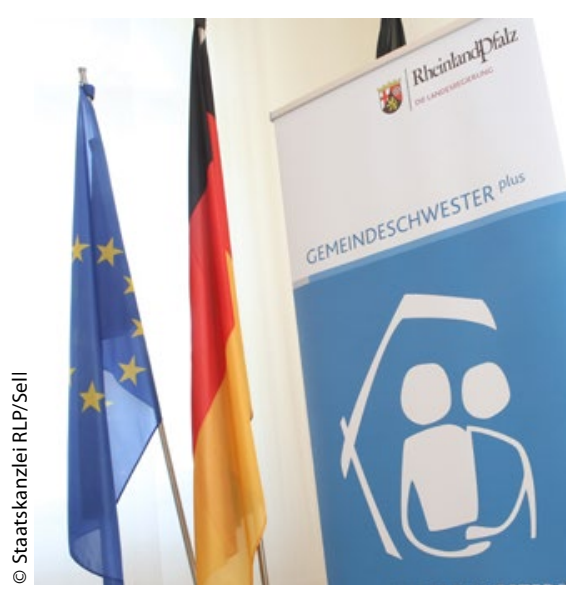

— In Rheinland-Pfalz startete im Juli mit dem Modellprojekt Gemeindeschwester plus ein neues Angebot für hochbetagte Menschen, die noch keine Pflege brauchen, sondern Unterstützung und Beratung in ihrem aktuellen Lebensabschnitt. Das Modellprojekt Gemeindeschwester ${ }^{\text {plus }}$ schließt eine Lücke bei der Unterstützung und Beratung, die von den Partnern im Gesundheitswesen und in der Pflege so - auch aufgrund leistungsrechtlicher Vorgaben - nicht geschlossen werden kann. Die Gemeinde- schwester ${ }^{\text {plus }}$ berät nicht zu Fragen der Pflege und erbringt keine Leistungen, die bereits heute von ambulanten Pflegediensten oder anderen Anbietern erbracht und von den Pflegekassen finanziert werden. Vielmehr wird sie Menschen nach deren vorheriger Zustimmung zuhause besuchen und sie individuell beraten: beispielsweise darüber, welche Angebote und sozialen Kontakte zur Stärkung der Selbstständigkeit zur Verfügung stehen und welche präventiven Vorkehrungen getroffen werden könnten, um Pflegebedürftigkeit möglichst lange zu vermeiden. Ziel ist, die Selbstständigkeit hochbetagter Menschen lange zu erhalten.

Nachdem aus insgesamt 22 Bewerbungen sechs Landkreise und drei kreisfreie Städte für das Projekt ausgewählt worden waren, startete der Bewerbungsprozess für die Pflegefachkräfte. Das Land fördert das Modell in dem Erprobungszeitraum von dreieinhalb Jahren zu 100\%. In der Implementierungsphase von Juli 2015 bis Dezember 2016 wird das Projekt wissenschaftlich eng begleitet.

www.gemeindeschwesterplus.rlp.de

\section{Rauchfrei durch die Pflegeausbildung}

— Jeder zweite Pflegeazubi raucht schon zu Beginn der Ausbildung. In der Altenpflege sind es sogar zwei von drei Auszubildenden. Das sind mehr als im Bevölkerungsdurchschnitt in der vergleichbaren Altersgruppe. Um gegen zu steuern fördert das Bundesministerium für Gesundheit seit 2013 zwei Modellprojekte zur Reduktion und Prävention des Tabakkonsums bei Pflegeazubis.

Das Projekt astra (Aktive Stressprävention durch Rauchfreiheit in der Pflege) arbeitet nach dem Modell des betrieblichen Gesundheitsmanagements und integriert Interventionen auf der Verhaltens- und Verhältnisebene. Es bringt die Interessen der Schule und der Praxis in einer gemeinsamen Steuerungsgruppe zusammen und umfasst fünf Module zur Situationsanalyse, Stressbewältigung, Rauchstoppangebot und Praxisprojekte zur Veränderung der Rahmenbedingungen in der Ausbildung. Das astra-Programm wurde in Kooperation mit dem Institut für Therapieforschung IFT München, der Hochschule Esslingen und dem Deutschen Netz Rauchfreier Krankenhäuser \& Gesundheitseinrichtungen DNRfK e.V. durchgeführt.

Das Projekt PA-TRES wurde an der Universität Würzburg entwickelt und bietet ein Unterrichtskonzept zu gesundem Lebensstil, Rauchen und Raucherberatung sowie Stressbewältigung in der Pflegeausbildung. Es umfasst zwölf Unterrichtseinheiten und wurde regional an zwölf Pflegeschulen erprobt.

www.astra-programm.de www.pa-tres.de 